\title{
THE DYNAMICS OF CONTINUOUS MAPS OF FINITE GRAPHS THROUGH INVERSE LIMITS
}

\author{
MARCY BARGE AND BEVERLY DIAMOND
}

\begin{abstract}
Suppose that $f: G \rightarrow G$ is a continuous piecewise monotone function on a finite graph $G$. Then the following are equivalent:

(i) $f$ has positive topological entropy;

(ii) there are disjoint intervals $I_{1}$ and $I_{2}$ and a positive integer $n$ with$$
I_{1} \cup I_{2} \subseteq f^{n}\left(I_{1}\right) \cap f^{n}\left(I_{2}\right) ;
$$

(iii) the inverse limit space constructed by using $f$ on $G$ as a single bonding map contains an indecomposable subcontinuum.

This result generalizes known results for the interval and circle.
\end{abstract}

\section{INTRODUCTION}

Suppose that $f: X \rightarrow X$ is a continuous function on a space $X$. One expects that certain dynamical properties of $f$ will be mirrored by topological properties of the inverse limit space $(X, f)$ with bonding map $f$, and vice versa. In particular, if $X$ is fairly simple and $f$ is dynamically complicated, $(X, f)$ should have a complicated topology.

A result along these lines is the following $\left[\mathrm{BM}_{1}\right]:$ if $f: I \rightarrow I$ is a piecewise monotone map of the interval $I$, then $f$ has a periodic point whose period is not a power of 2 if and only if the inverse limit space $(I, f)$ contains an indecomposable subcontinuum that is invariant under some power of the induced homeomorphism $\hat{f}:(X, f) \rightarrow(X, f)$. A similar theorem is proved for circle maps in [BR], and Roe proves in [Ro] that if $G$ is a finite graph and $f: G \rightarrow G$ has a certain periodic structure (depending on $G)$ then $(G, f)$ contains an indecomposable subcontinuum.

The main result of this paper generalizes the above to maps on finite graphs. One reason for our interest in inverse limits of maps on finite graphs is that they occur quite naturally as attractors for diffeomorphisms. R. F. Williams proved in [Wi] that if a manifold diffeomorphism $F$ has a one-dimensional hyperbolic attractor $\Lambda$ (with associated stable manifold structure) then $F$ restricted to $\Lambda$ is topologically conjugate with the induced homeomorphism $\hat{f}$ on an inverse limit of a piecewise monotone map $f$ of some finite graph. For certain $f$, Williams also proved a converse (in $S^{4}$ ) and it can be shown (along the lines of

Received by the editors May 27, 1992 and, in revised form, September 2, 1993.

1991 Mathematics Subject Classification. Primary 58F03, 58F13, 54H20.

Key words and phrases. Inverse limit, indecomposable continuum, finite graph, topological entropy, horseshoe. 
$\left[\mathrm{BM}_{2}\right]$ ) that for any map $f: G \rightarrow G$ of a finite graph there is a homeomorphism $F: R^{3} \rightarrow R^{3}$ with an attractor on which $F$ is conjugate with $\hat{f}$.

We prove

Main result. Suppose that $f: G \rightarrow G$ is a continuous piecewise monotone function on a finite graph $G$. The following are equivalent:

(i) the topological entropy of $f$ is positive;

(ii) the inverse limit space $(G, f)$ contains an indecomposable subcontinuum;

(iii) $f$ has a horseshoe; and

(iv) there are positive integers $r$ and $M$ such that for $m \geq M, f$ has a periodic point of prime period $\mathrm{rm}$.

Some of the implications are known and some hold without the hypothesis of piecewise monotonicity. The equivalence of (i) and (iv) for continuous functions on finite graphs is 3.2 of [Bl]. That (i) implies (iii) appears in [Mi] for continuous functions on the interval or circle. In recent work, Misiurewicz and Llibre have extended this result to maps on finite graphs by techniques different from ours. It is easy to see that (iii) implies (i) for any continuous function $f$ on any compact metric space $X$. That (ii) does not imply the other conditions in the absence of piecewise monotonicity, even for maps of the interval, is demonstrated by the pseudoarc example of Henderson $[\mathrm{H}]$. In case $G$ is an arc or a circle our theorem generalizes the previously mentioned results in $\left[\mathrm{BM}_{1}\right]$ and [BR] in that the indecomposable subcontinuum in the inverse limit is not required to be invariant under some power of the induced homeomorphism $\hat{f}$.

Note that if $f: G \rightarrow G$ is not onto, then $(G, f)=\left(G^{\prime}, f\right)$ where $G^{\prime}=$ $\bigcap_{n>0} f^{n}[G]$. Since $G^{\prime}$ is a finite graph, it suffices to prove the main result for surjections.

Let $f: G \rightarrow G$ be a continuous surjection on the finite graph $G$. The proof of the main result is as follows. In $\S 2$ we prove that (a) if $f$ has a horseshoe, then $(G, f)$ contains an indecomposable subcontinuum (2.4), and (b) if $f$ is piecewise monotone and $(G, f)$ contains an indecomposable subcontinuum, then $(G, f)$ contains an indecomposable subcontinuum that is invariant under $\hat{f}^{n}$ for some $n \geq 1$ (2.11). In $\S 3$ we show that if $f$ is piecewise monotone and $(G, f)$ is indecomposable, then $f$ has a horseshoe and thus has positive entropy (3.4). Combining the main results of $\S \S 2$ and 3, we conclude that, for piecewise monotone maps, if $(G, f)$ contains an indecomposable subcontinuum, then $f$ has a horseshoe (3.5). Finally, in $\S 4$ we show that if $f$ has positive entropy, then $(G, f)$ contains an indecomposable subcontinuum (4.3). In the remainder of this section we introduce some terminology and known results.

All functions are assumed continuous, and a map is a continuous surjection. A continuum $X$ is a compact, connected metric space, and is indecomposable if $X$ consists of more than one point and is not the union of two of its proper subcontinua. A continuum $X$ is irreducible between $x, y \in X$ if no proper subcontinuum of $X$ contains both $x$ and $y$; if $X$ is indecomposable, then there is an uncountable subset $S$ of $X$ such that $X$ is irreducible between any two points of $S$ (3-48 of [HY]). Given a continuum $X$ with metric $d$ and a continuous function $f: X \rightarrow X$, the associated inverse limit space $(X, f)$ is defined by

$$
(X, f)=\left\{\underline{x}=\left(x_{0}, x_{1}, \ldots\right) \mid x_{n} \in X, f\left(x_{n+1}\right)=x_{n} \text { for } n=0,1,2, \ldots\right\}
$$


with metric $\underline{d}$ given by

$$
\underline{d}(\underline{x}, \underline{y})=\sum_{n=0}^{\infty} \frac{d\left(x_{n}, y_{n}\right)}{2^{n}} .
$$

The space $(X, f)$ is a continuum, and the map $\hat{f}:(X, f) \rightarrow(X, f)$ defined by $\hat{f}\left(\left(x_{0}, x_{1}, \ldots\right)\right)=\left(f\left(x_{0}\right), x_{0}, x_{1}, \ldots\right)$ is called the induced homeomorphism. The projection maps $\Pi_{n}:(X, f) \rightarrow X$ given by $\Pi_{n}(\underline{x})=x_{n}$ are continuous; if $f$ is onto, the maps $\Pi_{n}$ are onto. If $H$ is a subcontinuum of $(X, f), H_{n}$ will denote $\Pi_{n}[H]$. Note that $f \circ \Pi_{n+1}=\Pi_{n}$ and $\Pi_{n}=\Pi_{n+1} \circ \hat{f}$.

A finite graph is a connected union of finitely many arcs. A map $f$ on a finite graph $G$ is piecewise monotone if there is a set $A=\left\{a_{1}, a_{2}, \ldots, a_{n}\right\}$ such that $f$ is monotone on each component of $G \backslash A$. That is, $\left(\left.f\right|_{C}\right)^{-1}(x)$ is connected for each $x \in G$ and each component $C$ of $G \backslash A$. Suppose that $f$ is piecewise monotone; for each $x \in G$, let $C_{x}$ denote the component of $f^{-1} f(x)$ containing $x$. Then $x$ is a turning point for $f$ if $f$ is not monotone on any neighbourhood of $C_{x}$ (i.e., if the graph of $f$ "folds" at $x$ ). Abusing standard terminology slightly, we say that $f$ has finitely many turning points if there is a finite set $\left\{x_{1}, \ldots, x_{n}\right\}$ such that no point of $G \backslash \bigcup_{i=1}^{n} C_{x_{i}}$ is a turning point for $f$. The function $f$ is piecewise monotone if and only if $f$ has finitely many turning points. A continuous function $f$ on the finite graph $G$ has a (one-dimensional) horseshoe if there are disjoint subcontinua $I_{0}$ and $I_{1}$ of $G$ and $n_{0} \in Z^{+}$such that $f^{n_{0}}\left(I_{0}\right) \supseteq I_{0} \cup I_{1}$ and $f^{n_{0}}\left(I_{1}\right) \supseteq I_{0} \cup I_{1}$ (where $Z^{+}$is the set of positive integers).

We make use of the following construction, which is a generalization of a much earlier construction for maps of the interval.

Theorem 1.1 [Ro]. Suppose that $f: G \rightarrow G$ is a continuous surjection and that $(G, f)$ contains no indecomposable subcontinuum with nonempty interior. Then there is an upper semicontinuous decomposition $\mathscr{G}$ of $(G, f)$ into disjoint subcontinua such that

(a) $\mathscr{G}$, with the quotient topology, is a finite graph;

if $g:(G, f) \rightarrow \mathscr{G}$ is the quotient map, then

(b) the subcontinuum $g(\underline{x})$ of $(G, f)$ has empty interior for each $\underline{x} \in$ $(G, f)$, and

(c) the map $f: \mathscr{G} \rightarrow \mathscr{G}$ defined by $F(g(\underline{x}))=g(\hat{f}(\underline{x}))$ is well defined and a homeomorphism.

As in (b) above, we shall think of $\xi \in \mathscr{G}$ both as an element of the space $\mathscr{G}$ and as a subcontinuum of $(G, f)$. The decomposition elements are precisely the maximal nowhere dense subcontinua of $(G, f)$. Equivalently, for $\underline{x} \in(G, f)$, the decomposition element containing $\underline{x}$ equals the intersection of all subcontinua of $(G, f)$ which contain in their interior a subcontinuum containing $\underline{x}$ in its interior.

\section{HORSESHOE $\rightarrow$ INDECOMPOSABLE SUBCONTINUUM}

Throughout this paper, $G$ will denote a finite graph, and $f: G \rightarrow G$ will be a continuous surjection on $G$.

In the first part of this section we prove that if $f: G \rightarrow G$ has a horseshoe, then $(G, f)$ contains an indecomposable subcontinuum (2.4). The map $f$ need not be piecewise monotone. 
Let $n$ and $l$ be positive integers. A continuum $X$ is called a $\Theta_{n, l}$ continuum provided: (i) for each subcontinuum $H$ of $X, X \backslash H$ has no more than $n$ components and (ii) if $L^{1}, \ldots, L^{l+1}$ are subcontinua of $X$ with $\bigcup_{i=1}^{l+1} L^{i} \neq \varnothing$, then, for some $i \in\{1, \ldots, l+1\}, L^{i} \subseteq \bigcup\left\{L^{j}: j \neq i, 1 \leq j \leq l+1\right\}$. It is evident that each finite graph is a $\Theta_{n, l}$ continuum for some $n, l \in N$. That this is also the case for an inverse limit space based on a finite graph is proved in $[R o]$. We require some minor extensions of this fact.

Lemma 2.1. If $H^{1}, H^{2}, \ldots, H^{m}$ are subcontinua of $(G, f)$, then

(i) $(G, f) \backslash \bigcup_{i=1}^{m} H^{i}$ has finitely many components, and

(ii) $H^{1} \cap H^{2}$ has finitely many components.

Proof. In case $m=1$, (i) is the result of 2.4 of [Ro]. Assume that (i) is true for $m \leq M$ and let $H^{1}, H^{2}, \ldots, H^{M+1}$ be subcontinua of $(G, f)$. Without loss of generality, $H^{i} \cap H^{j}=\varnothing$ for $i \neq j$. Then $H^{M+1}$ is contained in some component $C$ of $(G, f) \backslash \bigcup_{i=1}^{M} H^{i}$, hence $H^{M+1} \subseteq \mathrm{cl}(C)$. To prove the theorem it is enough to prove that $\operatorname{cl}(C) \backslash H^{M+1}$ has finitely many components.

Let $n$ and $l$ be such that $G$ is a $\Theta_{n, l}$ continuum and suppose that $V^{1}, \ldots$, $V^{l+1}$ are distinct components of $\operatorname{cl}(C) \backslash H^{M+1}$. Then $K^{i}=H^{M+1} \cup \operatorname{cl}\left(V^{i}\right)$, $i=1, \ldots, l+1$, are subcontinua of $(G, f)$ such that $\bigcup_{i=1}^{l+1} K^{i}=H^{n+1} \neq \varnothing$ and $K^{i} \not \subset \bigcup\left\{K^{j}: 1 \leq j \leq l+1, j \neq i\right\}$ for all $i$. It follows for sufficiently large $k$, the continua $\Pi_{k}\left[K^{i}\right], i=1, \ldots, l+1$, in $G$ satisfy: $\bigcap_{i=1}^{l+1} \Pi_{k}\left[K^{i}\right] \neq \varnothing$ for each $i, \Pi_{k}\left[K^{i}\right] \not \subset \bigcup\left\{\Pi_{k}\left[K^{j}\right]: 1 \leq j \leq l+1, j \neq i\right\}$. But this contradicts the choice of $l$ so that $\operatorname{cl}(C) \backslash H^{M+1}$ can have at most $l$ components.

To prove (ii), note that there is $s \in Z^{+}$such that the intersection of every pair of subcontinua of $G$ has no more than $s$ components. Suppose that $K^{1}, \ldots, K^{s+1}$ are distinct components of $H^{1} \cap H^{2}$. Then for all sufficient large $k, \Pi_{k}\left[K^{i}\right] \cap \Pi_{k}\left[K^{j}\right]=\varnothing$ for $i \neq j$. Since $\bigcup_{i=1}^{s+1} \Pi_{k}\left[K^{i}\right] \subseteq \Pi_{k}\left[H^{1} \cap H^{2}\right] \subseteq$ $\Pi_{k}\left[H^{1}\right] \cap \Pi_{k}\left[H^{2}\right]$ and $\Pi_{k}\left[H^{1}\right] \cap \Pi_{k}\left[H^{2}\right]$ has at most $s$ components, it must be the case that for some $i \neq j, \Pi_{k}\left[K^{i}\right]$ and $\Pi_{k}\left[K^{j}\right]$ are in the same component, say $J_{k}$, of $\Pi_{k}\left[H^{1}\right] \cap \Pi_{k}\left[H^{2}\right]$ for infinitely many $k$, say for $k_{1}<k_{2}<\cdots$. Then $f^{k_{i+1}-k_{i}}\left[J_{k_{i+1}}\right] \subseteq J_{k_{i}}$ for each $i$ and $J=\left\{\underline{x}: x_{k_{i}} \in J_{k_{i}}, i \in Z^{+}\right\}$is a subcontinuum of $H^{1} \cap H^{2}$ that contains both $K^{i}$ and $K^{j}$. Since this is not possible, $H^{1} \cap H^{2}$ has at most $s$ components.

The next result follows directly from 2.1 .

Lemma 2.2. Suppose that $(G, f)$ contains no indecomposable subcontinuum with nonempty interior. Then for each $m \geq 1$ there are nonempty subcontinua $H^{1}, H^{2}, \ldots, H^{m}$ of $(G, f)$ such that $(G, f)=\bigcup_{i=1}^{m} H^{i}$, and for each $i$, $\operatorname{int}\left(H^{i}\right)=(G, f) \backslash \bigcup_{j \neq i} H^{j}$.

Lemma 2.3. For any finite graph $G$, there is a positive integer $m$ so that if $L_{1}, L_{2}, \ldots, L_{m}$ are $m$ subcontinua of $G$ with $\bigcap_{1 \leq i \leq m} L_{i} \neq \varnothing$, then for some $i, L_{i} \subseteq \bigcup_{i \neq j} L_{j}$.

Theorem 2.4. Suppose that there are disjoint subcontinua $I_{0}$ and $I_{1}$ of $G$ and $n_{0} \in Z^{+}$such that $f^{n_{0}}\left(I_{0}\right) \supseteq I_{0} \cup I_{1}$ and $f^{n_{0}}\left(I_{1}\right) \supseteq I_{0} \cup I_{1}$. Then $(G, f)$ contains an indecomposable subcontinuum. 
Proof. Since the spaces $(G, f)$ and $\left(G, f^{n_{0}}\right)$ are homeomorphic, we need only show that if there are disjoint subcontinua $I_{0}$ and $I_{1}$ of $G$ such that $f\left(I_{0}\right) \supseteq$ $I_{0} \cup I_{1}$ and $f\left(I_{1}\right) \supseteq I_{0} \cup I_{1}$, then $(G, f)$ contains an indecomposable subcontinuum. Suppose that $I_{0}$ and $I_{1}$ are as above, and let $\Gamma=\operatorname{cl}\left(\bigcup_{n>0} f^{n}\left(I_{0} \cup I_{1}\right)\right)$. Then $\Gamma$ is a subcontinuum of $G$ and $f(\Gamma)=\Gamma$. We show that $(\Gamma, f)$ contains an indecomposable subcontinuum with nonempty interior.

Suppose not; let $m$ be as in 2.3 for $\Gamma$ and $l=\#\left[\operatorname{cl}\left(\Gamma \backslash I_{0}\right) \cap I_{0}\right]$. Choose $\left\{H^{1}, \ldots, H^{l m}\right\}$ as in 2.2. For each $i$ there is a nonempty open set $V^{i}$ of $\Gamma$ and $N_{i} \in Z^{+}$such that $\Pi_{N_{i}}^{-1}\left(V^{i}\right) \subseteq(\Gamma, f) \backslash\left(\bigcup_{j \neq i} H^{j}\right)=\operatorname{int}\left(H^{i}\right)$. Since $\Gamma=\operatorname{cl}\left(\cup_{n \geq 0} f^{n}\left(I_{0} \cup I_{1}\right)\right)$, for each $i$ there is $p_{i} \in I_{0} \cup I_{1}$ and $m_{i} \in Z^{+}$such that $f^{m_{i}}\left(p_{i}\right) \in V^{i}$. Then $\Pi_{N_{i}+m_{i}}^{-1}\left(p_{i}\right) \subseteq \operatorname{int}\left(H^{i}\right)$. Since $I_{0} \cup I_{1} \subseteq f\left(I_{0}\right)$, there is $\underline{x}^{i} \in \operatorname{int}\left(H^{i}\right)$ such that $x_{N_{i}+m_{i}}^{i}=p_{i}$ and $x_{N_{i}+m_{i}+k}^{i} \in I_{0}$ for $k \geq 1$.

Since $\underline{x}^{i} \in \operatorname{int}\left(H^{i}\right), \underline{x}^{i} \notin \bigcup_{j \neq i} H^{j}$. For each $i$, there is a positive integer $k_{i}$ so that if $k \geq k_{i}, x_{k}^{i} \notin \bigcup_{j \neq i} \Pi_{k}\left(H^{j}\right)$. Let

$$
N=\max \left(\left\{N_{i}+m_{i}+1: 1 \leq i \leq \operatorname{lm}\right\} \cup\left\{k_{i}: 1 \leq i \leq \operatorname{lm}\right\}\right) .
$$

Then for each $i$ :

(i) $\Pi_{N}\left(H^{i}\right) \cap I_{0} \neq \varnothing$, since $x_{N}^{i} \in I_{0}$;

(ii) $\Pi_{N}\left(H^{i}\right) \cap I_{1} \neq \varnothing$, by reasoning identical to that allowing the construction of $\underline{x}^{i}$; and

(iii) $x_{N}^{i} \in \Pi_{N}\left(H^{i}\right) \backslash \bigcup_{j \neq i} \Pi_{N}\left(H^{j}\right)$, since $N \geq k_{i}$.

It follows from (i) and (ii) that for each $i$, the subcontinuum $\Pi_{N}\left(H^{i}\right)$ contains at least one of the $l$ points of $\left[\operatorname{cl}\left(\Gamma \backslash I_{0}\right) \cap I_{0}\right]$. Since there are $l m$ of these subcontinua, at least $m$ of them have one point in common. But (iii) implies that no one of these subcontinua is contained in the union of others, contradicting the choice of $m$.

The remainder of this section is devoted to showing that if $f: G \rightarrow G$ is piecewise monotone and $(G, f)$ contains an indecomposable subcontinuum, then $(G, f)$ contains an indecomposable subcontinuum invariant under $\hat{f}^{n}$ for some $n \neq 0$.

Lemma 2.5. If $H$ is an indecomposable subcontinuum of $(G, f)$ with nonempty interior, then

(i) $\operatorname{int}(H)$ is connected, and

(ii) $H=\operatorname{cl}(\operatorname{int}(H))$.

Proof. Since $\operatorname{int}(H)=(G, f) \backslash \mathrm{cl}[(G, f) \backslash H]$, it follows from 2.1 that $\operatorname{int}(H)$ has finitely many components. Let $U$ be a component of $\operatorname{int}(H)$. Then $U$ is open, hence $\operatorname{cl}(U)$ is a subcontinuum of $H$ with nonempty interior. Since $H$ is indecomposable, $\operatorname{cl}(U)=H$; (i) and (ii) follow.

Lemma 8 of [BM] states that if $f$ is a continuous surjection on the unit interval $I$, and there is a proper subinterval $J$ of $I$ such that for all $n \in Z^{+}$, $f^{-n}[J]$ is an interval, then $(I, f)$ is decomposable. The proof of this result can easily be generalized to yield the following:

Lemma 2.6. Suppose that $f$ is a continuous surjection on the connected space $X$, and that $H$ is a subcontinuum of $(X, f)$. If there is a subcontinuum $J$ of $X$ such that for each $n \in Z^{+}, f^{-n}[J] \cap \Pi_{n}[H]$ is proper, connected, and has nonempty interior in $\Pi_{n}[H]$, then $H$ is decomposable. 
Lemma 2.7. Suppose that $f$ is piecewise monotone, and that $H$ is an indecomposable subcontinuum of $(G, f)$ with nonempty interior. Then there is an $n \neq 0$ such that $\hat{f}^{n}[H]=H$.

Proof. Since $H$ is indecomposable, it follows from 2.6 that for any positive integer $n$ and any proper subcontinuum $J$ of $\Pi_{n}[H]$, there is a positive integer $m$ such that $f^{-m}[J] \cap \Pi_{n+m}[H]$ is not connected. Then either $\Pi_{j}[H]$ contains a turning point of $f$ for infinitely many $j$, or $\Pi_{j}[H]$ contains a simple closed curve for infinitely many $j$. Since there are only finitely many turning points for $f$ and only finitely many simple closed curves, there is $p \in G$ and an infinite subset $B$ of $Z^{+}$such that $p \in \Pi_{n_{i}}[H]$ for $n_{i} \in B$.

Let $m$ be as in 2.3 for $G$. Choose $\underline{x} \in \operatorname{int}(H), N_{0} \in Z^{+}$such that $\Pi_{n}^{-1}\left[x_{n}\right] \subseteq \operatorname{int}(H)$ for $n \geq N_{0}$, and $n_{i} \geq N_{0}$. Since $\bigcup_{j=0}^{m-1} \Pi_{n_{i+j}}[H] \neq \varnothing$, there is $j \leq m-1$ such that $\Pi_{n_{i+j}}[H] \subseteq \bigcup\left\{\Pi_{n_{i+k}}[H]: 0 \leq k \leq m-1, k \neq j\right\}$. Now $\Pi_{n_{i+j}}^{-1}\left(x_{n_{i+j}}\right) \subseteq \operatorname{int}(H)$, hence $x_{n_{i+j}} \in \Pi_{n_{i+s}}[H]=\Pi_{n_{i+j}}\left[\hat{f}^{n_{i+j}-n_{i+s}}[H]\right]$ for some $s \in\{0,1, \ldots, m-1\} \backslash\{j\}$. It follows that $\hat{f}^{w}[H] \cap \operatorname{int}(H) \neq \varnothing$, where $w=n_{i+j}-n_{i+s}$.

Since $K=\hat{f}^{w}[H]$ is indecomposable, $K=\operatorname{cl}(\operatorname{int}(K))$, and so $\operatorname{int}(K)=$ $\operatorname{int}(H) \neq \varnothing$. It follows from 2.1 that $K \cap H$ has finitely many components, so at least one, say $C$, has nonempty interior. Since $H$ and $K$ are indecomposable, $C=H$ and $C=K$. That is, $\hat{f}^{w}[H]=H$.

Recall that a point $x$ is recurrent under $f$ if there is a sequence $n_{i} \rightarrow \infty$ such that $f^{n_{i}}(x) \rightarrow x$.

Lemma 2.8. If $h$ is a homeomorphism of the finite graph $G$ and $h$ has a recurrent nonperiodic point, then $G$ is homeomorphic to the circle and $h$ has no periodic points.

Proof. Suppose that the set $B$ of branch points of $G$ is nonempty. Then $B$ is finite and invariant under the homeomorphism $h$, hence if $x \in G$ is recurrent and nonperiodic, $x \in A$ for some component $A$ of $G \backslash B$. If $n$ is the smallest positive integer such that $h^{n}[A]=A$, then $x$ is recurrent under $\left.h^{n}\right|_{A}$. But $A$ is an arc, and all recurrent points of a homeomorphism of an arc are periodic. Thus $B=\varnothing$, and $G$ is either an arc or a circle. According to the above, $G$ is a circle. If $h$ has any periodic point of period $k$, then for each $x \in G, h^{2 n k}(x)$ converges to a periodic point. But then the only recurrent points are periodic, and the lemma is proved.

Lemma 2.9. Suppose that $A \subseteq G$ is an arc containing no branch points. If $A \subseteq f[A]$ but $A \not \subset \operatorname{int}(f[A])$ then $f$ has a fixed point in $A$.

Proof. Let $A=[a, b]$. Without loss of generality, $a \notin \operatorname{int}(f[A])$. Let $x_{1}=$ $\inf \{x \in A: f(x)=a\}$.

Suppose that $b \in f\left[a, x_{1}\right]$, and let $x_{2}=\sup \left\{x \in\left[a, x_{1}\right]: f(x)=b\right\}$. If $f\left[x_{2}, x_{1}\right]$ contains a point of $G \backslash A$, then either $a \in \operatorname{int}(f[A])$, or there is $c \in\left(x_{2}, x_{1}\right)$ such that $c=f(b)$, and so $f\left[x_{2}, x_{1}\right]=[a, b]$. That is, $\left[x_{2}, x_{1}\right] \subseteq f\left[x_{2}, x_{1}\right]$, and $f$ has a fixed point.

Similarly, if $b \in f\left[x_{1}, b\right]$, and $x_{2}=\inf \left\{x \in\left[x_{1}, b\right]: f(x)=b\right\}$, then $\left[x_{1}, x_{2}\right] \subseteq f\left[x_{1}, x_{2}\right]$, and $f$ has a fixed point. 
Lemma 2.10. Suppose that $H$ is an indecomposable subcontinuum of $(G, f)$, and that $(G, f)$ contains no indecomposable subcontinuum with nonempty interior. Let $\mathscr{G}$ be the decomposition space of $(G, f)$ with induced homeomorphism $F: \mathscr{G} \rightarrow \mathscr{G}$. If $\xi=g(H)$ is the decomposition element of $\mathscr{G}$ containing $H$, then $\xi$ is periodic under $F$.

Proof. We first prove that $\xi$ is recurrent under $F$. Suppose not; there is then an open neighbourhood $U$ of $\xi$ such that $F^{-n}(\xi) \subseteq C=\mathscr{G} \backslash U$ for all $n \geq 1$. Since $H \subseteq g^{-1}(\xi)=\xi, H$ and $g^{-1}[C]$ are disjoint compact subsets of $(G, f)$ and $\hat{f}^{-n}[H] \subseteq g^{-1}[C]$ for all $n \geq 1$. There is a positive integer $N_{0}$ such that if $k \geq N_{0}$ and $n \geq 1, \Pi_{k}[H] \cap \Pi_{k+n}[H]=\Pi_{k}[H] \cap \Pi_{k}\left[\hat{f}^{-n}[H]\right] \subseteq \Pi_{k}[H] \cap$ $\Pi_{k}\left[g^{-1}[C]\right]=\varnothing$. Then the projections $\Pi_{k}[H]$, for $k \geq N_{0}$, are pairwise disjoint. But this contradicts the fact that, since $H$ is indecomposable, there is, as in the proof of 2.7, an infinite subset $B$ of $Z^{+}$such that $\bigcap_{n_{i} \in B} \Pi_{n_{i}}[H] \neq \varnothing$. Therefore $\xi$ is recurrent.

If $\xi$ is not periodic under $F$, then according to $2.8, \mathscr{G}$ must be a circle and $F$ has no periodic points. Suppose that for every $n \in Z^{+}, \Pi_{n}(\xi)$ contains either a loop or a branch point. If $k=(\#$ of branch points of $G)+(\#$ of loops in $G)+1$, then for each $n \in Z^{+}$, there are $i(n), j(n) \in\{1, \ldots, k\}$ with $i(n) \neq j(n)$ and $\Pi_{n k+i(n)}(\xi) \cap \Pi_{n k+j(n)}(\xi) \neq \varnothing$. Choose $i, j \in\{i, \ldots, k\}$ such that $i \neq j$ and $i=i(n)$ and $j=j(n)$ for infinitely many $n$. Let $s=j-i$; then $\Pi_{N+n k+i}(\xi) \cap \Pi_{N+n k+j}(\xi)=\Pi_{N+n k+j}\left[\hat{f}^{s}(\xi)\right] \cap \Pi_{N+n k+j}(\xi) \neq \varnothing$ for infinitely many $n$. It follows that $\xi \cap \hat{f}^{s}(\xi) \neq \varnothing$, hence $\xi=\hat{f}^{s}(\xi)$ and $\xi$ is periodic. So assume that for some positive integer $r, \Pi_{r}(\xi)$ is an arc containing no branch points.

Suppose that the infinite set $B$ has been chosen so that $\varnothing \neq \bigcap_{n_{i} \in B} \Pi_{n_{i}}[H] \subseteq$ $\bigcap_{n_{i} \in B} \Pi_{n_{i}}(\xi)$. Let $m$ be as in 2.3 for $G$, and choose $n_{s} \in B$ so that $n_{s} \geq r$. For some $i \in\{0, \ldots, m-1\}$,

$$
\Pi_{n_{s+i}}(\xi) \subseteq \bigcup\left\{\Pi_{n_{s+j}}(\xi): 0 \leq j \leq m-1, i \neq j\right\} .
$$

Let $l=n_{s+i}-r$. Then

$$
\begin{aligned}
\Pi_{r}(\xi) & =f^{l}\left[\Pi_{n_{s+i}}(\xi)\right] \subseteq \bigcup\left\{f^{l}\left[\Pi_{n_{s+j}}(\xi)\right]: 0 \leq j \leq m-1, i \neq j\right\} \\
& =\bigcup\left\{\left[\Pi_{n_{s+j}-l}(\xi)\right]: 0 \leq j \leq m-1, i \neq j\right\} .
\end{aligned}
$$

(If $k<0$, let $\Pi_{k}(\xi)=f^{|k|}\left[\Pi_{0}(\xi)\right]$.)

We consider two cases.

Case 1. $\Pi_{r}(\xi) \not \subset \operatorname{int}\left[\bigcup\left\{\Pi_{n_{s+j}-l}(\xi): 0 \leq j \leq m-1, i \neq j\right\}\right]$.

Since $\Pi_{r}(\xi)$ is an arc containing no branch points, there is an integer $t=$ $n_{s+j}-l$ for some $j \in\{0, \ldots, k-1\}, j \neq i$, such that either $\Pi_{r}(\xi) \subseteq \Pi_{t}(\xi)$ (but $\Pi_{r}(\xi) \not \subset \operatorname{int}\left(\Pi_{t}(\xi)\right)$ or $\Pi_{t}(\xi) \subseteq \Pi_{r}(\xi)$. Note that $t \neq r$.

(1a) If $\Pi_{r}(\xi) \subseteq \Pi_{t}(\xi)$ and $t>r$, then $f^{t-r}\left[\Pi_{t}(\xi)\right]=\Pi_{r}(\xi)$, so $f^{t-r}\left[\Pi_{r}(\xi)\right] \subseteq$ $\Pi_{r}(\xi)$ and $f$ has a periodic point in $G$. Then $\hat{f}$ has a periodic point in $(G, f)$ and $F$ has a periodic point in $\mathscr{G}$. But $F$ has no periodic points.

(1b) If $\Pi_{r}(\xi) \subseteq \Pi_{t}(\xi)$ and $t<r$, then $f^{r-t}\left[\Pi_{r}(\xi)\right]=\Pi_{t}(\xi) \supseteq \Pi_{r}(\xi)$. Since $\Pi_{r}(\xi) \not \subset \operatorname{int}\left(f^{r-t}\left[\Pi_{r}(\xi)\right]\right), 2.9$ implies that $f^{r-t}$ has a fixed point, and $f$ has a periodic point. As in (1a), we are done.

(1c) If $\Pi_{t}(\xi) \subseteq \Pi_{r}(\xi)$ and $r>t$, then $f^{r-t}\left[\Pi_{r}(\xi)\right]=\Pi_{t}(\xi) \subseteq \Pi_{r}(\xi)$, and $f$ has a periodic point. 
(1d) If $\Pi_{t}(\xi) \subseteq \Pi_{r}(\xi)$ and $r<t$, then $f^{t-r}\left[\Pi_{t}(\xi)\right]=\Pi_{r}(\xi)$ and $\Pi_{t}(\xi)$ is a subarc of $\Pi_{r}(\xi)$. Hence $f$ has a periodic point.

Case 2. $\Pi_{r}(\xi) \subseteq \operatorname{int}\left[\bigcup\left\{\Pi_{n_{s+j}-l}(\xi): 0 \leq j \leq m-1, i \neq j\right\}\right]$. Since $\Pi_{r}(\xi)$ is an arc containing no branch points, and since $\bigcap_{j=0}^{m-1} \Pi_{n_{s+j}-l}(\xi) \neq \varnothing$, there are $t_{1}, t_{2} \in\left\{n_{s+j}-l: 0 \leq j \leq m-1, j \neq i\right\}$ with $t_{1} \neq t_{2}$ and such that $\Pi_{r}(\xi) \subseteq \operatorname{int}\left[\Pi_{t_{1}}(\xi) \cup \Pi_{t_{2}}(\xi)\right]$. Then $\xi \subseteq \Pi_{r}^{-1}\left(\operatorname{int}\left[\Pi_{t_{1}}(\xi) \cup \Pi_{t_{2}}(\xi)\right]\right)$. Since the decomposition $\mathscr{G}$ is upper semicontinuous, there is an arc $I \subseteq \mathscr{G}$ such that $\xi \in I$, and for $\eta \in \operatorname{int}(I), \Pi_{r}(\eta) \subseteq \operatorname{int}\left[\Pi_{t_{1}}(\xi) \cup \Pi_{t_{2}}(\xi)\right]$.

Since $F$ is a circle homeomorphism without periodic points, $\xi$ is recurrent and $\xi \in \operatorname{int}(I)$, there is a positive integer $N$ such that $\bigcup_{i=0}^{N-1} F^{i}[I]=\mathscr{G}$. (To see that this is the case, let $n>0$ be such that $F^{n}(\xi) \in I$ and let $G^{\sim}: \mathbf{R} \rightarrow \mathbf{R}$ be a lift of $F^{n}$ such that $G^{\sim}\left(I^{\sim}\right) \cap I^{\sim} \neq \varnothing$ where $I^{\sim}$ is a component of $\Pi^{-1}(I)$. Let $\xi^{\sim} \in \Pi^{-1}(I)$. Since $F$ has no periodic points, $\left\{F^{k n}\left(\xi^{\sim}\right) \mid k=1,2, \ldots\right\}$ is unbounded, and hence for sufficiently large $m$, the arc $\bigcup_{k=0}^{m} F^{k n}\left(I^{\sim}\right)$ contains a fundamental domain. Let $N=n m+1$.)

Since $\bigcup_{i=0}^{N-1} F^{i}[I]=\mathscr{G}, \Pi_{r}\left[\bigcup_{i=0}^{N-1} F^{i}[I]\right]=G$, and so $\Pi_{r}\left[\bigcup_{i=0}^{N-1} \hat{f}^{i}[I]\right]=G$. But then $\bigcup_{i=0}^{N-1} f^{i}\left[\Pi_{r}[I]\right]=G$. Since $\Pi_{r}[I] \subseteq \Pi_{t_{1}}(\xi) \cup \Pi_{t_{2}}(\xi)$,

$$
\bigcup_{i=0}^{N-1} f^{i}\left[\Pi_{t_{1}}(\xi) \cup \Pi_{t_{2}}(\xi)\right]=G .
$$

Without loss of generality $t_{2}>t_{1}$, so that $f^{t_{2}-t_{1}}\left[\Pi_{t_{2}}(\xi)\right]=\Pi_{t_{1}}(\xi)$. Then $\bigcup_{i=0}^{N-1} f^{i}\left[f^{t_{2}-t_{1}}\left[\Pi_{t_{2}}(\xi)\right] \cup \Pi_{t_{2}}(\xi)\right]=G$, and so $\bigcup_{i=0}^{N-1}\left[\Pi_{t_{2}} \hat{f}^{i}\left[\hat{f}^{t_{2}-t_{1}}(\xi)\right] \cup \Pi_{t_{2}}\left(\hat{f}^{i}(\xi)\right)\right]$ $=G$. For $i=0$ to $N-1$, let $\eta_{i}=\hat{f}^{i}(\xi)$ and $\eta_{N-1+i}=\hat{f}^{i}\left[\hat{f}^{t_{2}-t_{2}}(\xi)\right]$. Then the $2 N$ elements $\eta_{0}, \eta_{1}, \ldots, \eta_{2 N-1}$ have the property that $\Pi_{t_{2}}\left[\bigcup_{i=0}^{2 N-1} \eta_{i}\right]=G$. If $t_{2} \geq 0$, then $\Pi_{0}\left[\bigcup_{i=0}^{2 N-1} \eta_{i}\right]=G$. If $t_{2}<0$, then $\Pi_{0}\left[\bigcup_{i=0}^{2 N-1} \hat{f}^{-t_{2}}\left(\eta_{i}\right)\right]=G$. In any case there are $2 N$ elements of $\mathscr{G}$, say $\eta_{0}, \eta_{1}, \ldots, \eta_{2 N-1}$, whose union projects under $\Pi_{0}$ onto $G$. It follows that for any $n \geq 0, \Pi_{k}\left[\bigcup_{i=0}^{2 N-1} \hat{f}^{n}\left(\eta_{i}\right)\right]=$ $G$ for $0 \leq k \leq n$. That is, for $n \geq 0$, for any $x \in(G, f)$, there is $i \in$ $\{0,1, \ldots, 2 N-1\}$ such that $\underline{d}\left(\underline{x}, \hat{f}^{n}\left(\eta_{i}\right)\right)<1 / 2^{n}$.

Choose $\left\{U_{i}\right\}_{i=0}^{2 N}$ to be a collection of disjoint open intervals in $\mathscr{G}$ with centers $\left\{a_{i}\right\}_{i=0}^{2 N}$. Choose $\underline{x}^{i} \in g^{-1}\left(a_{i}\right)$, and let $\varepsilon=\min \left\{d\left(\underline{x}^{i},(G, f) \backslash g^{-1}\left(U_{i}\right)\right)\right\}_{i=0}^{2 N}$. For any $n \geq 0$, for some $i \in\{0, \ldots, 2 N\}$,

$$
U_{i} \cap\left\{F^{n}\left(\eta_{0}\right), F^{n}\left(\eta_{1}\right), \ldots, F^{n}\left(\eta_{2 N-1}\right)\right\}=\varnothing
$$

and thus $g^{-1}\left(U_{i}\right) \cap\left[\bigcup_{j=0}^{2 N-1} \hat{f}^{n}\left(\eta_{j}\right)\right]=\varnothing$. But then for every $n \geq 0$, for some $i=$ $\{0, \ldots, 2 N\}, \underline{d}\left(\underline{x}^{i}, \bigcup_{j=0}^{2 N-1} \hat{f}^{n}\left(\eta_{j}\right)\right) \geq \varepsilon$, a contradiction to the last paragraph.

It follows that $\xi$ must be periodic under $F$.

Note that the only property required of $\xi$ in the proof of 2.10 is that there is an infinite set $B$ such that $\bigcap_{n_{i} \in B} \Pi_{n_{i}}[\xi] \neq \varnothing$.

Theorem 2.11. Suppose that $f: G \rightarrow G$ is piecewise monotone. If $(G, f)$ contains an indecomposable subcontinuum then $(G, f)$ contains an indecomposable subcontinuum that is invariant under $\hat{f}^{n}$ for some $n \neq 0$.

Proof. Let $H$ be an indecomposable subcontinuum of $(G, f)$. As in the proof of 2.7 , there is an infinite subset $B$ of $Z^{+}$such that $\bigcap_{n_{i} \in B} \Pi_{n_{i}}[H] \neq \varnothing$. Let 
$L_{1}$ be a subcontinuum of $(G, f)$ irreducible with respect to the properties: $\hat{f}\left[L_{1}\right]=L_{1}$ and $\hat{f}^{n}[H] \subseteq L_{1}$ for all $n \in Z$.

Let $f_{1}=\left.f\right|_{L_{1}}$ and $G_{1}=\Pi_{0}\left[L_{1}\right]$. Suppose that $L_{1}=\left(G_{1}, f_{1}\right)$ does not contain an indecomposable subcontinuum with nonempty interior; otherwise by 2.7 the theorem is proved. Let $\mathscr{G}_{1}=\left\{g_{1}(\underline{x}): x \in L_{1}\right\}$ be the decomposition space of $\left(G_{1}, f_{1}\right)$ with quotient map $g_{1}$ and let $F_{1}: \mathscr{G}_{1} \rightarrow \mathscr{G}_{1}$ be the induced homeomorphism. Let $\xi_{1}=g_{1}(\underline{x})$ for $\underline{x} \in H$.

According to $2.10, \xi_{1}$ is periodic under $F_{1}$. If $\xi_{1}$ were fixed by $F_{1}$, then since $\mathscr{G}_{1}$ is nondegenerate, $\xi_{1}$ would be a proper subcontinuum of $L_{1}$ that is invariant under $\hat{f}$ and contains $\hat{f}^{n}[H]$ for all $n \in Z$, contradicting the definition of $L_{1}$. Then $\xi_{1}$ is of least period $k_{1}>1$ under $F_{1}$, and $\hat{f}_{1}^{i}\left(\xi_{1}\right) \cap$ $\hat{f}_{i}^{j}\left(\xi_{1}\right)=\varnothing$ for $i, j \in\left\{0, \ldots, k_{1}-1\right\}, i \neq j$. It follows that the projections $\Pi_{0}\left(\xi_{1}\right), \ldots, \Pi_{k_{1}-1}\left(\xi_{1}\right)$ are pairwise disjoint. Hence $\Pi_{0}(H), \ldots, \Pi_{k_{1}-1}(H)$ are pairwise disjoint.

Let $L_{1} \subseteq \xi_{1}$ be a subcontinuum irreducible with respect to the properties: $\hat{f}^{k_{1}}\left[L_{2}\right]=L_{2}$ and $\hat{f}^{n k_{1}}[H] \subseteq L_{2}$ for all $n \in Z$. Let $G_{2}=\Pi_{0}\left[L_{2}\right], f_{2}=$ $\left.f_{1}^{k_{1}}\right|_{G_{2}}: G_{2} \rightarrow G_{2}$, and $H^{2}=\left\{\underline{x} \in\left(G_{2}, f_{2}\right): x_{n} \in \Pi_{n k_{1}}[H]\right.$ for $\left.n \in Z^{+}\right\}$. Then $H^{2}$ is homeomorphic to $H$ and hence is indecomposable. If $L_{2}=\left(G_{2}, f_{2}\right)$ contains an indecomposable subcontinuum with nonempty interior, the theorem holds. Otherwise, let $\mathscr{G}_{2}$ be the decomposition space of $\left(G_{2}, f_{2}\right)$, with induced homeomorphism $F_{2}: \mathscr{G}_{2} \rightarrow \mathscr{G}_{2}$, and let $\xi_{2}=g_{2}(\underline{x})$ for $x \in H^{2}$. As before, $\xi_{2}$ must be periodic under $F_{2}$ of least period $k_{2}>1$. It follows that the projections $\Pi_{0}\left(\xi_{2}\right), \Pi_{0}\left[\hat{f}_{2}^{-1}\left(\xi_{2}\right)\right], \ldots, \Pi_{0}\left[\hat{f}_{2}^{1-k_{2}}\left(\xi_{2}\right)\right]$ are pairwise disjoint, and thus that $\Pi_{0}\left(\xi_{2}^{\prime}\right), \Pi_{0}\left(\hat{f}_{1}^{-1}\left(\xi_{2}^{\prime}\right)\right], \ldots, \Pi_{0}\left[\hat{f}_{1}^{1-k_{1} k_{2}}\left(\xi_{2}^{\prime}\right)\right]$ are pairwise disjoint, where $\xi_{2}^{\prime}=$ $\left\{\underline{x} \in\left(G_{1}, f_{1}\right) \mid x_{n k_{1}} \in \Pi_{n}\left(\xi_{2}\right)\right.$ for $\left.n \geq 0\right\}$. Then $\Pi_{0}(H), \ldots, \Pi_{k_{1} k_{2}-1}(H)$ are pairwise disjoint.

Continuing, either we find an indecomposable continuum invariant under $\hat{f}^{n}$ for some $n \neq 0$, or we construct a sequence of integers $\left\{k_{i}\right\}_{i \in Z^{+}}$with $k_{i}>1$ for all $i$ and such that $\Pi_{0}(H), \Pi_{1}(H), \ldots, \Pi_{k_{1} k_{2} \cdots k_{s}-1}(H)$ are pairwise disjoint for all $s$. Since this contradicts the fact that $\bigcap_{n_{i} \in B} \Pi_{n_{i}}[H] \neq \varnothing$, there is an indecomposable subcontinuum of $(G, f)$ invariant under $\hat{f}^{n}$ for some $n \neq 0$.

\section{INDECOMPOSABLE SUBCONTINUUM $\rightarrow$ HORSESHORE}

In this section we prove that if $f$ is piecewise monotone, and $(G, f)$ contains an indecomposable subcontinuum, then $f$ has a horseshoe (3.5). As indicated in the introduction, the result is not true without the hypothesis of piecewise monotonicity.

The following is proved in $[\mathrm{Ku}]$; we include a proof for the sake of completeness.

A subset $H$ of $G$ is $\varepsilon$-dense in $G$ if for each $x \in G, d(x, H)<\varepsilon$, where $d(x, H)$ is in terms of minimal arc length.

Lemma 3.1. Suppose that $(G, f)$ is irreducible between $\underline{a}$ and $\underline{b}$ and that $\left\{J_{n}\right\}_{n \in Z^{+}}$is a sequence of subcontinua of $G$ such that for all $n,\left\{a_{n}, b_{n}\right\} \subseteq J_{n}$. Then $\lim _{n \rightarrow \infty} f^{n}\left(J_{n}\right)=G$ (in the Hausdorff metric).

That is, for $\varepsilon>0$, there is $N_{0} \in Z^{+}$such that for all $n \geq N_{0}, f^{n}\left(J_{n}\right)$ is $\varepsilon$-dense in $G$. 
Proof. Suppose that $\left\{J_{n}\right\}_{n \in Z^{+}}$is such a sequence, and that $\lim _{n \rightarrow \infty} f^{n}\left(J_{n}\right) \neq$ $G$. There is then an infinite subset $B_{0}$ of $Z^{+}$and a proper subcontinuum $J_{0}^{\prime}$ of $G$ such that $\left\{f^{n_{i}}\left(J_{n_{i}}\right): n_{i} \in B_{0}\right\}$ converges to $J_{0}^{\prime}$. Certainly $\left\{a_{0}, b_{0}\right\} \subseteq$ $J_{0}^{\prime}$. There is a second infinite subset $B_{1}$ of $B_{0}$ and a subcontinuum $J_{1}^{\prime}$ such that $\left\{f^{n_{i}-1}\left(J_{n_{i}}\right): n_{i} \in B_{1}\right\}$ converges to $J_{1}^{\prime}$. Then $f\left[J_{1}^{\prime}\right]=J_{0}^{\prime}$, and $\left\{a_{1}, b_{1}\right\} \subseteq J_{1}^{\prime}$. Continuing in this way, we get a sequence of subcontinua $\left\{J_{n}^{\prime}: n \in Z^{+}\right\}$with $f\left[J_{n+1}^{\prime}\right]=J_{n}^{\prime}$, and $\left\{a_{n}, b_{n}\right\} \subseteq J_{n}^{\prime}$ for all $n \in Z^{+}$. Define $J=\left\{\left(z_{0}, z_{1}, \ldots\right) \mid z_{n} \in J_{n}^{\prime}, z_{n}=f\left(z_{n+1}\right)\right\}$. Then $J$ is a proper subcontinuum of $(G, f)$ containing $\underline{a}$ and $\underline{b}$, contradicting the fact that $(G, f)$ is irreducible between $\underline{a}$ and $\underline{b}$.

We need a slight generalization of the notion of horseshoe: for $n \geq 2$, the map $f$ has an $n$-horseshoe if there are disjoint intervals $I_{1}, I_{2}, \ldots, I_{n}$ and a positive integer $n_{0}$ such that $f^{n_{0}}\left[I_{j}\right] \supseteq \bigcup_{i=1}^{n} I_{i}$ for $1 \leq j \leq n$.

Lemma 3.2. Suppose that $I_{1}, I_{2}, \ldots, I_{n}$ are disjoint intervals in $G$.

(a) If $n \geq 3$ and there is $n_{0} \in Z^{+}$such that each of $I_{1}, I_{2}, \ldots, I_{n}$ maps under $f^{n_{0}}$ over at least $n-1$ of $I_{1}, I_{2}, \ldots, I_{n}$, then $f$ has an $(n-1)$-horseshoe.

(b) If $n \geq 2$ and there are positive integers $k_{1}, k_{2}, \ldots, k_{n}$ such that for each $j, f^{k j}\left[I_{j}\right] \supseteq \bigcup_{i=1}^{n} I_{i}$, then $f$ has an n-horseshoe.

Proof. (a) Let $A$ be the $n \times n$ matrix with $i j$ th entry 1 if $I_{i}$ maps over $I_{j}$ under $f^{n_{0}}$ and 0 otherwise. Then the $i j$ th entry of $A^{2}$ is nonzero if and only if $I_{i}$ maps over $I_{j}$ under $f^{2 n_{0}}$. Suppose that for some $i$ and $j$, the $i j$ th entry of $A^{2}$ is zero. Since each row of $A$ contains at most one zero entry, the $j$ th column of $A$ contains at most one nonzero entry. Suppose that $a_{k j}=1$ for some $k \neq i$. Then $a_{i j}=0$, hence $a_{i m}=1$ for $m \neq j$. In particular, $a_{i k} a_{k j}=1$ and the $i j$ th entry of $A^{2}$ is nonzero. Then $a_{k j}=0$ for $k \neq i$, hence $a_{k m}=1$ for $k \neq i, m \neq j$. Let $B$ denote the $(n-1) \times(n-1)$ matrix obtained by deleting the $j$ th row and $j$ th column of $B$. Every entry of $B$ is nonzero except for possibly one zero in the row corresponding to the original $i$ th row of $A$. But then every entry of $B^{2}$ is nonzero. That is, for $k=1$ to $n, k \neq j, f^{2 n_{0}}\left[I_{k}\right] \supseteq \bigcup\left\{I_{k}: k=1\right.$ to $\left.n, k \neq j\right\}$.

(b) Let $w=\sum_{i=1}^{n} k_{i}$. For each $i, f^{w}\left[I_{i}\right]=f^{w-k_{i}}\left[f^{k_{i}}\left[I_{i}\right]\right] \supseteq f^{w-k_{i}}\left[\bigcup_{i=1}^{n} I_{i}\right] \supseteq$ $f^{w-k_{i}}\left[I_{j}\right]$ (for some $\left.j \neq i\right) \supseteq f^{w-k_{i}-k_{j}}\left[f^{k_{j}}\left[I_{j}\right]\right] \supseteq f^{w-k_{i}-k_{j}}\left[\bigcup_{i=1}^{n} I_{i}\right] \supseteq \cdots \supseteq$ $\bigcup_{i=1}^{n} I_{i}$.

Lemma 3.3. Suppose that $(G, f)$ is indecomposable. Let $F=\left\{x \in G:\left|f^{-n}(x)\right|\right.$ $=1$ for all $n \geq 0$. Then

(i) $F$ is a finite set, so that if $a \in F$ then $a$ is periodic.

(ii) Suppose that $f$ is piecewise monotone and let $n_{0}$ be the period of $a \in F$. There is $m>0$ and a base $\mathscr{V}$ of neighbourhoods of a such that for $V \in \mathscr{V}$, $f^{m n_{0}}[V] \subseteq V$.

Proof. (i) Choose $\left\{\underline{x}^{i}\right\}_{i=1}^{6} \subseteq(G, f)$ so that for a fixed edge $E$ and an infinite subset $B$ of positive integers, $\left\{x_{n_{j}}^{i}\right\}_{i=1}^{6} \subseteq E$ for each $n_{j} \in B$, and such that $(G, f)$ is irreducible between $\underline{x}^{i}$ and $\underline{x}^{k}$ if $i \neq k$. Since $\underline{x}^{i} \neq \underline{x}^{k}$ if $i \neq k$, we can assume that for $n_{j} \in B, x_{n_{j}}^{i} \neq x_{n_{j}}^{k}$. We can also assume that an orientation of $E$ is fixed and that for $n_{j} \in B,\left\{x_{n_{j}}^{i}\right\}_{i=1}^{6}$ appears in the order $x_{n_{j}}^{1}<x_{n_{j}}^{2}<\cdots<x_{n_{j}}^{6}$, since otherwise some ordering appears infinitely often and we pass to a subsequence and relabelling. 
Suppose that two distinct elements $a_{1}$ and $a_{2}$ of $F$ lie in the same edge $E^{\prime}$ of $G$. Choose $\varepsilon>0$ to be smaller than the minimum length of a minimal path from $a_{1}$ to $a_{2}$. According to 3.2 , there is a positive integer $N_{0}$ so that for $n_{j} \in B, n_{j} \geq N_{0}$ and $i=1$ to $5, f^{n_{j}}\left[x_{n_{j}}^{i}, x_{n_{j}}^{i+1}\right]$ is $\varepsilon$-dense in $G$. Then one of $a_{1}$ and $a_{2}$ is in $f^{n_{j}}\left[x_{n_{j}}^{i}, x_{n_{j}}^{i+1}\right]$ for each $i$, and thus one of $a_{1}$ and $a_{2}$ is in $f^{n_{j}}\left[x_{n_{j}}^{i}, x_{n_{j}}^{i+1}\right]$ for at least three $i$-values. But at least two of the three corresponding intervals $\left[x_{n_{j}}^{i}, x_{n_{j}}^{i+1}\right]$ are disjoint, contradicting the fact that $\left|f^{-n_{j}}\left(a_{1}\right)\right|=1=\left|f^{-n_{j}}\left(a_{2}\right)\right|$. Then $|F| \leq \#$ of edges of $G<\aleph_{0}$. It follows that if $a \in F$ then the backwards orbit of $a$ is finite, hence $a$ is periodic with period $n_{0}$ no larger than the number of edges of $G$.

(ii) Suppose that $a \in F$ has period $n_{0}$. Since $\left|f^{-n}(a)\right|=1$ for $n>0$ and $f^{n_{0}}$ has finitely many turning points, a connected neighbourhood $V$ of $a$ can be chosen so that $V$ contains no turning point of $f^{n_{0}}$ and thus $f^{n_{0}}$ is $1-1$ on $V$. Then $m$ can be chosen so that for each edge $E$ extending from $a, f^{m n_{0}}$ maps some initial segment of $E$ into $E$.

Let $g=f^{n m_{0}}$, and fix an edge $E$ extending from $a$. Since $\left|g^{-1}(a)\right|=1$ and $g$ has finitely many turning points, there is $c \in E$ such that $\left|g^{-1}(x)\right|=1$ for all $x \in[a, c]$. Suppose that $c$ can be chosen so that for all $x \in[a, c],[a, x] \subseteq$ $g[a, x]$. Then $g^{-1}[a, x] \subseteq[a, x]$, from which it follows that $g^{-n}[a, x]$ is an interval for all $n \in Z^{+}$. According to 2.6, this contradicts the indecomposability of $(G, f)$. Then given $c$ above, there is $x_{1} \in[a, c]$ with $g\left[a, x_{1}\right] \subseteq\left[a, x_{1}\right]$. The neighbourhood of $a$ is constructed in the obvious way.

Since the point $x_{1}$ used to construct the neighbourhood of $a$ can be taken as close to $a$ as desired, $a$ has a base of such neighbourhoods.

The following theorem can be strengthened to prove that $f$ has an $n$-horseshoe for any $n$, a fact we need later. Since the proof of 3.4 is somewhat technical, we first prove the existence of a 2 -horseshoe, and then indicate the details of the generalization (3.4b).

Theorem 3.4. Suppose that $f$ is piecewise monotone and that $(G, f)$ is indecomposable. Then $f$ has a horseshoe.

Proof. Choose a set $K$ consisting of 26 distinct elements of $(G, f)$ with the property that there is a fixed edge $E$ of $G$ and an infinite subset $A$ of $Z^{+}$such that for $n_{i} \in A,\left\{x_{n_{j}}^{i}: 1 \leq i \leq 26\right\} \subseteq E$, and such that $(G, f)$ is irreducible between any two elements of $K$.

Claim 1. Either (i) there is a subset $\left\{\underline{z}^{i}\right\}_{i=1}^{6}$ of $K$ and an infinite subset $B_{1}$ of $A$ such that for $i \in\{1, \ldots, 6\},\left\{z_{n_{j}}^{i}: n_{j} \in B_{1}\right\}$ converges to a single point $z_{i}$, and $z_{i} \neq z_{j}$ if $i \neq j$, or

(ii) there is a subset $\left\{\underline{x}^{i}\right\}_{i=1}^{6}$ of $K$ and an infinite subset $B_{2}$ of $A$ such that for $i=\{1, \ldots, 6\},\left\{x_{n_{j}}^{i}: n_{j} \in B_{2}\right\}$ converges to the same point $x$.

Proof of Claim 1. Choose $\underline{x}^{1}$ arbitrarily from $K$, and an infinite subset $B_{1}^{1}$ of $A$ such that $\left\{x_{n_{j}}^{1}: n_{j} \in B_{1}^{1}\right\}$ converges to a single point $x_{1}$. Either at least five elements of $K$ have the property that $\left\{x_{n_{j}}: n_{j} \in B_{1}^{1}\right\}$ converges to $x_{1}$ also, or for at least 21 of the elements of $K,\left\{x_{n_{j}}: n_{j} \in B_{1}^{1}\right\}$ has a limit point different than $x_{1}$. In the latter case, choose $x^{2}$ arbitrarily from this set of 21 , and $B_{1}^{2} \subseteq B_{1}^{1}$ to be infinite and such that $\left\{x_{n_{j}}^{\frac{2}{2}}: n_{j} \in B_{1}^{2}\right\}$ converges to a single point $x_{2} \neq x_{1}$. Continuing in this fashion, we get at least one of the two sets described above, and the claim is proved. 
Suppose that (i) occurs. As in the proof of 3.3(i), we can assume that for $n_{j} \in B_{1}, z_{n_{j}}^{i} \neq z_{n_{j}}^{k}$. We can also assume that an orientation of $E$ is fixed and that for $n_{j} \in B_{1},\left\{z_{n_{j}}^{i}\right\}_{i=1}^{6}$ appears in the order $z_{n_{j}}^{1}<z_{n_{j}}^{2}<\cdots<z_{n_{j}}^{6}$.

Define $\varepsilon=\min \left\{d\left(z_{i}, z_{k}\right): i \neq k ; i, k \in\{1, \ldots, 6\}\right\}$. According to 3.1, there is a positive integer $N_{0}$ such that if $n_{j} \geq N_{0}$ and $i \in\{1, \ldots, 5\}$, then $f^{n_{j}}\left[z_{n_{j}}^{i}, z_{n_{j}}^{i+1}\right]$ is $\varepsilon / 2$-dense in $G$. In addition, $N_{0}$ can be chosen large enough so that if $n_{j} \geq N_{0}$ and $i \in\{1, \ldots, 6\}, d\left(z_{i}, z_{n_{j}}^{i}\right)<\varepsilon / 4$. Then for $n_{j} \geq N_{0}$, the sets $\left\{\left[z_{n_{j}}^{i}, z_{n_{j}}^{i+1}\right]\right\}_{i=1,3,5}$ are pairwise disjoint intervals, and each maps under $f^{n_{j}}$ over at least two of the three. It follows from 3.2 that $f$ has a horseshoe.

Suppose then that (ii) occurs. Again we assume that for $n_{j} \in B_{2},\left\{x_{n_{j}}^{i}\right\}_{i=1}^{6}$ appears in the order $x_{n_{j}}^{1}<x_{n_{j}}^{2}<\cdots<x_{n_{j}}^{6}$.

Claim 2. There is an infinite set $B_{3} \subseteq B_{2}$ so that for at least one $i$-value $i_{0}$, the collection $\left\{\left[x_{m_{j}}^{i_{0}}, x_{m_{j}}^{i_{0}+1}\right]\right\}_{m_{j} \in B_{3}}$ is an infinite collection of pairwise disjoint intervals.

Proof of Claim 2. For each $n_{j} \in B_{2}$ and $i=1$ to 5, the limit point $x$ can be in at most two of the intervals $\left[x_{n_{j}}^{i}, x_{n_{j}}^{i+1}\right]$. It follows that for at least three $i$-values, $x \notin\left[x_{n_{j}}^{i}, x_{n_{j}}^{i+1}\right]$ for infinitely many $n_{j}$ 's in $B_{2}$. Let $i_{0}$ be an $i$-value for which this is the case and choose $m_{1}$ to be the smallest element of $B_{2}$ with $x \notin\left[x_{m_{1}}^{i_{0}}, x_{m_{1}}^{i_{0}+1}\right]$. Suppose that $m_{1}<m_{2}<\cdots<m_{k}$ have been inductively chosen so that $\left\{\left[x_{m_{j}}^{i_{0}}, x_{m_{j}}^{i_{0}+1}\right]\right\}_{j=1}^{k}$ is a pairwise disjoint collection of intervals and $x \notin \bigcup_{j=1}^{k}\left\{\left[x_{m_{j}}^{i_{0}}, x_{m_{j}}^{i_{0}+1}\right]\right\}$. Let $\delta_{k}=\min \left\{d\left(x, x_{m_{k}}^{i_{0}}\right), d\left(x, x_{m_{k}}^{i_{0}+1}\right)\right\}$. Choose $N_{k}$ so that if $n_{j} \geq N_{k}$ and $n_{j} \in B_{2}$, then $d\left(x, x_{n_{j}}^{i}\right)<\delta_{k}$ for $i=1$ to 6 . There is a least element $m_{k+1}$ of $B_{2}$ satisfying $m_{k+1} \geq N_{k}$ and $x \notin\left[x_{m_{k+1}}^{i_{0}}, x_{m_{k+1}}^{i_{0} 1}\right]$. Then $\left[x_{m_{k+1}}^{i_{0}}, x_{m_{k+1}}^{i_{0}+1}\right] \cap\left[\bigcup_{j=1}^{k}\left[x_{m_{j}}^{i_{0}}, x_{m_{j}}^{i_{0}+1}\right]\right]=\varnothing$, and $x \notin \bigcup_{j=1}^{k+1}\left[x_{m_{j}}^{i_{0}}, x_{m_{j}}^{i_{0}+1}\right]$.

Let $B_{3}=\left\{m_{k}\right\}_{k \in Z^{+}}$defined above; Claim 2 is proved.

Claim 3. Suppose there are $m_{1}, m_{2}, n_{1}, n_{2} \in B_{3}$ such that $n_{1} \neq n_{2}$, $m_{i} \neq n_{j}$ for $i, j \in\{1,2\}$ and

$$
\left[x_{m_{j}}^{i_{0}}, x_{m_{j}}^{i_{0}+1}\right] \not \subset f^{n_{j}-m_{j}}\left[x_{n_{j}}^{i_{0}}, x_{n_{j}}^{i_{0}+1}\right] \quad \text { for } j=1,2
$$

(where $i_{0}$ is as in Claim 2). Then $f$ has a horseshoe.

Proof of Claim 3. Let $I_{m_{k}}=\left[x_{m_{k}}^{i_{0}}, x_{m_{k}}^{i_{0}+1}\right]$ for all $m_{k} \in B_{3}$. Since $f^{n_{j}-m_{j}}\left(x_{n_{j}}^{i_{0}}\right)$ $=x_{m_{j}}^{i_{0}}$ for $j=1,2, f^{n_{j}-m_{j}}\left[I_{n_{j}}\right] \supseteq E \backslash I_{m_{j}}$. In particular, for $j=1,2, I_{n_{1}} \cup$ $I_{n_{2}} \subseteq f^{n_{j}-m_{j}}\left[I_{n_{j}}\right]$. It follows from 3.2(b) that $f$ has a horseshoe and Claim 3 holds.

Recall that for $m_{j} \in B_{3},\left\{x_{m_{j}}^{i}\right\}_{i=1}^{6}$ is contained in the fixed edge $E$. According to Claim 3, if $f$ does not have a horseshoe, there is $m_{j} \in B_{3}$ such that for $k \geq i \geq j, I_{m_{i}} \subseteq f^{m_{k}-m_{i}}\left[I_{m_{k}}\right]$. Without loss of generality this holds for all $m_{i}, m_{k} \in B_{3}$.

Claim 4. Suppose that there is $n \in Z^{+}$and $m_{j} \in B_{3}$ such that $E \subseteq$ $f^{n}\left[x_{m_{j}}^{i_{0}}, x_{m_{j}}^{i_{0}+1}\right]$. Then $f$ has a horseshoe.

Proof of Claim 4. Again let $I_{m_{k}}=\left[x_{m_{k}}^{i_{0}}, x_{m_{k}}^{i_{0}+1}\right]$. According to Claim 3, we can assume that $f^{m_{j+1}-m_{j}}\left[I_{m_{j+1}}\right] \supseteq I_{m_{j}}$ and $f^{m_{j+2}-m_{j}}\left[I_{m_{j+2}}\right] \supseteq I_{m_{j}}$. But then $E \subseteq f^{m_{j+1}-m_{j}+n}\left[I_{m_{j+1}}\right]$, and $E \subseteq f^{m_{j+2}-m_{j}+n}\left[I_{m_{j+2}}\right]$. It follows from 3.2(b) that $f$ has a horseshoe. 
Claim 5. There is $a \in E$ with $\left|f^{-n}(a)\right|=1$ for all $n \in Z^{+}$.

Proof of Claim 5. For each $m_{k} \in B_{3}$ define

$$
J_{m_{k}}=\bigcup_{m_{j} \geq m_{k}, m_{j} \in B_{3}} f^{m_{j}-m_{k}}\left[x_{m_{j}}^{i_{0}}, x_{m_{j}}^{i_{0}+1}\right] .
$$

Then $J_{m_{k}}$ is connected and so $\operatorname{cl}\left(J_{m_{k}}\right)$ is a subcontinuum of $G$.

For $n \in Z^{+}$, let $m_{k}$ be the least element of $B_{3}$ such that $n \leq m_{k}$ and let $J_{n}=f^{m_{k}-n}\left[J_{m_{k}}\right]$. Then $\operatorname{cl}\left(J_{n}\right)$ is a subcontinuum of $G$, and $f\left[J_{n+1}\right]=$ $J_{n}$. Define $J=\left\{\left(z_{0}, z_{1}, \ldots\right) \mid z_{n} \in \operatorname{cl}\left(J_{n}\right), z_{n}=f\left(z_{n+1}\right)\right\}$. Then $J$ is a subcontinuum of $(G, f)$ containing $\left\{\underline{x}^{i}\right\}_{i=1}^{6}$, thus $J=(G, f)$. Since $f$ is onto, $\Pi_{n}[J]=\operatorname{cl}\left(J_{n}\right)=G$.

Suppose that for some $n, J_{n} \supseteq E$. Let $m_{k}$ be the smallest element of $B_{3}$ with $m_{k} \geq n$. Then $f^{m_{k}-n}\left[J_{m_{k}}\right]=J_{n} \supseteq E$. But $J_{m_{k}}$ is the union of nested continua so there is $m_{j} \in B_{3}$ with $m_{j} \geq m_{k}$ and $f^{m_{k}-n}\left[f^{m_{j}-m_{k}}\left[x_{m_{j}}^{i_{0}}, x_{m_{j}}^{i_{0}+1}\right]\right] \supseteq$ $E$. But then $f^{m_{j}-n}\left[x_{m_{j}}^{i_{0}}, x_{m_{j}}^{i_{0}+1}\right] \supset E$, contradicting the choice of $i_{0}$. So $E \not \subset J_{n}$ for any $n$. But $J_{n}$ is connected and $\operatorname{cl}\left(J_{n}\right)=G$, so for each $n$ there is a set $F_{n}=G \backslash J_{n}$ with $\left|F_{n}\right| \leq k$, where $k$ is the cardinality of the largest discrete set that can be removed from $G$ without disconnecting the remaining subgraph. Since $f\left[J_{n+1}\right]=J_{n}, f^{-1}\left[F_{n}\right] \subseteq F_{n+1}$, and so $\left|F_{n}\right| \leq\left|F_{n+1}\right| \leq k$. Then there is a positive integer $N_{0}$ so that if $n \geq N_{0},\left|F_{n}\right|=\left|F_{n+1}\right|$. That is, for $n \geq N_{0}$ and $a \in F_{n},\left|f^{-1}(a)\right|=1$, thus $\left|f^{-k}(a)\right|=1$ for $k \geq 0$. Since $F_{n} \cap E \neq \varnothing$, Claim 5 holds.

According to the proof of 3.3(i), $a$ is periodic and $f^{-n}(a) \cap E \subseteq\{a\}$ for all $n \geq 0$.

Claim 6. For $1 \leq i \leq 6$ and $n \in Z^{+}, x_{m_{j}}^{i} \neq a$.

Proof of Claim 6. Suppose that there are $j \leq 6$ and $n \in Z^{+}$such that $x_{n}^{j}=a$. Since $f^{-n}(a) \cap E \subseteq\{a\}$ for all $n \in Z^{+}, x_{m_{k}}^{i}=a$ for any $m_{k} \in B_{3}$. It follows that if $i \neq j, x_{n}^{i} \neq a$ for any $n \in Z^{+}$, for otherwise $\underline{x}^{j}=\underline{x}^{i}$. Let $n_{0}$ be the period of $a$, and choose $m \in Z^{+}$and $V$ an open neighbourhood of $a$ such that $f^{m n_{0}}[V] \subseteq V$. According to 3.3, the set $V$ can be chosen small enough so that $V \cap\left\{x_{k}^{i}: i=1\right.$ to $\left.6, i \neq j, 1 \leq k \leq m n_{0}\right\}=\varnothing$. Then for $i \neq j$, $x_{m_{k}}^{i} \notin V$ for $m_{k} \in B_{3}$. Recall that for each $i,\left\{x_{m_{k}}^{i}: m_{k} \in B_{3}\right\}$ converges to the same limit point $x$. Apparently $x=a$, and thus there is a positive integer $N_{0}$ so that $\left\{x_{m_{k}}^{i}: m_{k} \in B_{3}, m_{j} \geq N_{0}, i \leq 6\right\} \subseteq V$. This is a contradiction to the construction of $V$, and Claim 6 holds.

Then the open neighbourhood $V$ of $a$ can be chosen so that $f^{m n_{0}}[V] \subseteq V$ and $V \cap\left\{x_{k}^{i}: i=1\right.$ to $\left.6,1 \leq k \leq m n_{0}\right\}=\varnothing$. As above, $x_{m_{j}}^{i} \notin V$ for $m_{j} \in B_{3}$ and $i \leq 6$. That is, for each $m_{j} \in B_{3},\left[x_{m_{j}}^{i}, x_{m_{j}}^{i+1}\right] \subseteq E \backslash V$ except for possibly one $i$-value, and for that $i$-value $V \subseteq\left[x_{m_{j}}^{i}, x_{m_{j}}^{i+1}\right]$.

Choose $\varepsilon>0$ so that the $\varepsilon$-ball centered at $a$ is contained in $V$, and choose $N_{1}$ large enough so that for $i=1$ to $5, m_{j} \in B_{3}$ and $m_{j} \geq N_{1}, f^{m_{j}}\left[x_{m_{j}}^{i}, x_{m_{j}}^{i+1}\right]$ is $\varepsilon$-dense in $G$. For a fixed $m_{j}$, the interiors of the intervals $\left[x_{m_{j}}^{i}, x_{m_{j}}^{i+1}\right]_{i+1}^{5}$ are disjoint, hence $a \in f^{m_{j}}\left[x_{m_{j}}^{i}, x_{m_{j}}^{i+1}\right]$ for at most one $i$-value. For the remaining four $i$-values, $E \backslash V \subseteq f^{m_{j}}\left[x_{m_{j}}^{i}, x_{m_{j}}^{i+1}\right]$, and according to the previous paragraph, for at least three of these, $\left[x_{m_{j}}^{i}, x_{m_{j}}^{i+1}\right] \subseteq E \backslash V$. Since at least two of the three intervals $\left[x_{m_{j}}^{i}, x_{m_{j}}^{i+1}\right]$ are disjoint, $f$ has a horseshoe. 
For maps of the interval, the existence of an $n$-horseshoe follows easily from the existence of a 2-horseshoe-given an interval $I$ mapping over intervals $J$ and $K$, there are disjoint subintervals $I_{1}$ and $I_{2}$ of $I$ mapping over $J$ and $K$ respectively, etc. As is clear from the above proof, the latter statement need not hold for maps on circles or finite graphs, thus the existence of $n$-horseshoes and the "monotone" horseshoes of 3.5 requires some details.

Theorem 3.4b. Suppose that $f$ is piecewise monotone and that $(G, f)$ is indecomposable. Then $f$ has an $n$-horseshoe for any positive integer $n$.

Proof. We trace the proof of 3.4 to ensure that at each point that a horseshoe is produced, an $n$-horseshoe can be produced. First, it is clear that the size of the original irreducible set can be as large as desired, and the cases (i) and (ii) in the first paragraph can be witnessed by sets of any finite size. Case (i) follows immediately from $3.2(\mathrm{a})$.

The appropriate generalization of Claim 3 is the following:

Claim 3b. Suppose that for the positive integer $n$, there are disjoint subsets $\left\{m_{i}: 1 \leq i \leq n\right\}$ and $\left\{n_{i}: 1 \leq i \leq n, n_{j} \neq n_{i}\right.$ for $\left.i \neq j\right\}$ of $B_{3}$ such that for each $i$,

$$
\left[x_{m_{j}}^{i_{0}}, x_{m_{j}}^{i_{0}+1}\right] \not \subset f^{n_{i}-m_{i}}\left[x_{n_{i}}^{i_{0}}, x_{n_{i}}^{i_{0}+1}\right] .
$$

Then $f$ has an $n$-horseshoe.

As with Claim 3, this follows from 3.2(b).

Then if $f$ does not have an $n$-horseshoe, there is $m_{j} \in B_{3}$ such that for $k \geq i \geq j, E \backslash I_{m_{i}} \subseteq f^{m_{k}-m_{i}}\left[I_{m_{k}}\right]$. Without loss of generality this holds for all $m_{i}, m_{k} \in B_{3}$.

Claim 4b. Suppose that there is $n_{0} \in Z^{+}$and $m_{j} \in B_{4}$ such that $E \subseteq$ $f^{n_{0}}\left[x_{m_{j}}^{i_{0}}, x_{m_{j}}^{i_{0}+1}\right]$. Then $f$ has an $n$-horseshoe.

Proof of Claim 4b. Again let $I_{m_{k}}=\left[x_{m_{k}}^{i_{0}}, x_{m_{k}}^{i_{0}+1}\right]$. As in the proof of 3.4, $f^{m_{j+i}-m_{j}+n_{0}}\left[I_{m_{j+i}}\right] \supseteq E$ for $1 \leq i \leq n$, and Claim $4 \mathrm{~b}$ follows from $3.2(\mathrm{~b})$.

The proofs of Claims 5 and 6 need no changes, and the neighbourhood $V$ of the fixed point $a$ can be constructed so that for some fixed $m_{j}$ and as large a set of $i$-values as desired, $\left[x_{m_{j}}^{i}, x_{m_{j}}^{i+1}\right] \subseteq E \backslash V \subseteq f^{m_{j}}\left[x_{m_{j}}^{i}, x_{m_{j}}^{i+1}\right]$. Then $f$ has an $n$-horseshoe.

Note that the horseshoe constructed in 3.4 or $3.4 \mathrm{~b}$ has all intervals contained in a single edge without branch points.

An interval $I$ maps monotonely over the interval $J$ under $f$ if there is a subinterval $K$ of $I$ such that $f[K]=J$. A horseshoe $\left\{I_{1}, I_{2}\right\}$ for $f$ is monotone with respect to $f^{n}$ if for $i, j=1,2$, there are subintervals $I_{i j}$ of $I_{i}$ such that $f^{n}\left[I_{i j}\right]=I_{j}$.

Corollary 3.5. Suppose that $f$ is piecewise monotone and $(G, f)$ contains an indecomposable subcontinuum. Then $f$ has a monotone horseshoe.

Proof. Since $(G, f)$ contains an indecomposable subcontinuum, $(G, f)$ contains an indecomposable subcontinuum $H$ invariant under $\hat{f}^{n}$ for some $n \in$ $Z^{+}$. The inverse limit spaces $(G, f)$ and $\left(G, f^{n}\right)$ are homeomorphic; let $H_{1}$ be the image of $H$ under the natural homeomorphism, and let $J=\Pi_{0}\left[H_{1}\right]$. Then $J$ is invariant under $f^{n}$, and $g=\left.f^{n}\right|_{j}$ is piecewise monotone, so according to $3.4 \mathrm{~b}, g$ has a 3-horseshoe $\left\{I_{1}, I_{2}, I_{3}\right\}$ with $I_{1} \cup I_{2} \cup I_{3} \subseteq E$ for some edge $E$ of $J$ having no branch points. Then each of $I_{i}$ maps monotonely under $g^{m}$ 
over at least two of $I_{1}, I_{2}$ and $I_{3}$, where $m$ is the positive integer witnessing the horseshoe. Since the property of mapping monotonely (under some iterate) is transitive, arguments identical to those in the proof of 3.2 imply that at least two of $I_{1}, I_{2}, I_{3}$ form a monotone horseshoe with respect to $g^{2 m}$. But this monotone horseshoe will also be a monotone horseshoe for $f$ on $G$.

We can now deduce a "stability" result for piecewise monotone functions on finite graphs.

Corollary 3.6. Suppose that $f: G \rightarrow G$ is piecewise monotone and that $(G, f)$ contains an indecomposable subcontinuum. If $g$ is any continuous map on $G$ sufficiently close to $f,(G, g)$ contains an indecomposable subcontinuum.

Proof. According to 3.5, $f$ has a horseshoe $\left\{I_{1}, I_{2}\right\}$ monotone with respect to $f^{n}$ for some positive integer $n$. By using the monotonicity of the horseshoe repeatedly, one can find four subintervals $J_{1}, J_{2}, J_{3}, J_{4}$ of $I_{1}$ such that $f^{3 n}\left[J_{i}\right]=I_{1}$ for $i=1$ to 4 . For some $\varepsilon>0$ and $i$-values $i_{1}, i_{2}$, the distance from $J_{i j}$ to the endpoints of $I_{1}$ is larger than $\varepsilon$ for $j=1,2$. Without loss of generality, if an edge $E^{\prime}$ of $G$ is not contained in $I_{1}$, there is $x \in E^{\prime}$ such that $d\left(x, I_{1}\right) \geq \varepsilon$, where $d\left(x, I_{1}\right)$ refers to minimal arc length. Suppose that $d\left(f^{3 n}, g^{3 n}\right)<\varepsilon$. Since $f^{3 n}\left[J_{i_{j}}\right]=I_{1}, g^{3 n}\left[J_{i_{j}}\right] \supseteq J_{i_{1}} \cup J_{i_{2}}$ for $j=1,2$. That is, $g$ has a horseshoe. According to 2.5, $(G, g)$ contains an indecomposable subcontinuum.

\section{Positive ENTROPY $\rightarrow$ INDECOMPOSABLE SUBCONTINUUM}

Suppose that $f: X \rightarrow X$ is a map of the compact metric space $X$ with metric $d$. For $n \in N$ and $\varepsilon>0$, a set $E \subseteq X$ is $(n, \varepsilon)$-separated under $f$ provided that for distinct $x, y \in E$, there is a $k$ such that $0 \leq k<n$ and $d\left(f^{k}(x), f^{k}(y)\right)>\varepsilon$. Given $A \subseteq X$, let $E(n, \varepsilon, A)$ be a subset of $A$ of maximal cardinality that is $(n, \varepsilon)$-separated under $f$. Let $h(f, A, \varepsilon)=$ $\limsup \operatorname{su}_{n \rightarrow \infty}(\log \# E(n, \varepsilon, A)) / n$, and $h(f, A)=\lim _{\varepsilon \rightarrow 0} h(f, A, \varepsilon)$. The topological entropy of $f$ is then $h(f)=h(f, X)$. (There are several equivalent definitions of entropy; see, for example, [AKM] or [Bo].) We review here a few facts about entropy:

(i) $h(f)$ is independent of (equivalent) metric;

(ii) for $n \geq 1, h\left(f^{n}\right)=n h(f)$;

(iii) if $f$ is a homeomorphism, $h\left(f^{-1}\right)=h(f)$;

(iv) if $\hat{f}:(X, f) \rightarrow(X, f)$ is the induced homeomorphism then $h(\hat{f})=$ $h(f)$;

(v) if $f: G \rightarrow G$ is a homeomorphism of a finite graph $G$ then $h(f)=0$;

(vi) if $f: X \rightarrow X$ and $g: Y \rightarrow Y$ are maps of the compact spaces $X$ and $Y$ and $p: Y \rightarrow X$ is a continuous surjection such that $f \circ p=p \circ g$, then $h(g) \leq h(f)+\sup _{x \in X} h\left(\left.g\right|_{p^{-1}(x)}\right)$ and

(vii) $h(f)=h(f, \Omega(f))$, where $\Omega(f)=\{x \in X \mid$ for each neighbourhood $U$ of $x$ there is $n \geq 1$ such that $\left.f^{n}(U) \cap U \neq \varnothing\right\}$ is the nonwandering set of $f$.

Of the above, (i), (ii), (iii), (vi) and (vii) can be found in [Bo]; (iv) is in [G] and [Ba]; (v) follows from (ii) and the like results in case $G$ is an arc or circle [AKM]. 
In the following, $\mathscr{G}$ will denote the decomposition space of Theorem 1.1 associated with $(X, f)$ with induced homeomorphism $F: \mathscr{G} \rightarrow \mathscr{G}$.

Lemma 4.1. If $h(f)>0$ and $(G, f)$ contains no indecomposable subcontinuum with nonempty interior, then there is $\xi \in \mathscr{G}$ that is periodic under $F$ and for which $h\left(\hat{f}, g^{-1}(\xi)\right)>0$.

Proof. We have $0<h(f)=h(\hat{f})=h\left(\hat{f}^{-1}\right) \leq h\left(F^{-1}\right)+\sup _{\xi \in \mathscr{G}} h\left(\hat{f}^{-1}, g^{-1}(\xi)\right)$ by (iii), (iv) and (vi) above. Since $h\left(F^{-1}\right)=0((\mathrm{v})$ above), there is $\xi \in \mathscr{G}$ such that $h\left(\hat{f}^{-1}, g^{-1}(\xi)\right)>0$.

If it were that $\lim _{k \rightarrow \infty} \operatorname{diam}\left(\Pi_{k}\left[g^{-1}(\xi)\right]\right)=0$, then

$$
\lim _{n \rightarrow \infty} \operatorname{diam}\left(\hat{f}^{-n}\left[g^{-1}(\xi)\right]\right)=0
$$

and it would follow that $h\left(\hat{f}^{-1}, g^{-1}(\xi)\right)=0$. Thus $\lim _{k \rightarrow \infty} \operatorname{diam}\left(\Pi_{k}\left[g^{-1}(\xi)\right]\right)$ $\neq 0$, and there is a $p \in G$ such that $p \in \Pi_{n}\left[g^{-1}(\xi)\right]$ for infinitely many $n$. According to the remark following $2.10, \xi$ is periodic under $F$, say of period $n$. Then $0<h\left(\hat{f}^{-1}, g^{-1}(\xi)\right)=(1 / n) h\left(\left.\hat{f}^{-n}\right|_{g^{-1}(\xi)}\right)=(1 / n) h\left(\left.\hat{f}^{n}\right|_{g^{-1}(\xi)}\right)=$ $h\left(\hat{f}, g^{-1}(\xi)\right)$.

By an $n$-od we will mean a space which is the union of $n$ arcs $A_{1}, A_{2}, \ldots$, $A_{n}$ and which contains a point $p$ such that $A_{i} \cap A_{j}=\{p\}$ for $i \neq j$. It is clear that if $f: X \rightarrow X$ is a homeomorphism of the $n$-od $X$ and $x$ is a periodic point of $f$ then the period of $x$ divides $n$ !.

Lemma 4.2. Suppose that $f: X \rightarrow X$ is a map of the $n$-od $X$ and that $h(f)>$ 0 . Then $(X, f)$ contains an indecomposable subcontinuum.

Proof. According to 3.2 of [Bl], if $h(f)>0$ there are positive integers $r$ and $M$ such that $\operatorname{Per}(f) \equiv\{m \mid f$ has a periodic point of period $m\} \supseteq\{m r \mid m \geq M\}$. In particular, $f$ must have a periodic point, say $x$, of period $p r$ where $p$ is a prime larger than $n !$. Let $\underline{x}$ be a corresponding point in $(X, f)$ of period $p r$ under $\hat{f}$.

Suppose that $(X, f)$ is hereditary decomposable. It is a consequence of Theorem A of [Ro] that $\mathscr{G}$ is a tree with no more than $n$ endpoints, hence the period of every periodic point of $F$ divides $n$ !. In particular, if $\xi \in \mathscr{G}$ contains $\underline{x}$ then the period $n_{1}$ of $\xi$ divides both $n$ ! and $p r$. Since $p>n$ ! and $p$ is prime, $n_{1}$ must divide $r$.

Let $X_{1}=\Pi_{0}\left[g^{-1}(\xi)\right]$, where $g:(X, f) \rightarrow \mathscr{G}$ is the quotient map, and let $f_{1}=\left.f^{n_{1}}\right|_{X_{1}}: X_{1} \rightarrow X_{1}$. Then $\left(X_{1}, f\right)$ contains a periodic point of period $p\left(r / n_{1}\right)$ under $f_{1}$ and $X_{1}$ is an $l_{1}$-od for some $l_{1} \leq n$. Let $\mathscr{G}_{2}$ be the decomposition space of $\left(X_{1}, f_{1}\right)$ and let $F_{2}: \mathscr{G}_{2} \rightarrow \mathscr{G}_{2}$ be the homeomorphism induced by $\hat{f}_{1}$ (note that $\left(X_{1}, f_{1}\right)$ is homeomorphic with the subcontinuum $g^{-1}(\xi)$ of $(X, f)$ and thus is hereditarily decomposable). Let $\xi_{2} \in \mathscr{G}_{2}$ contain a point of period $p\left(r / n_{1}\right)$ under $\hat{f}_{1}$. Then, as above, the period $n_{2}$ of $\xi_{2}$ under $F_{2}$ divides $r / n_{1}$.

Proceeding in this manner, we define a strictly decreasing sequence $X_{k}$ of $l_{k}$-ods, $l_{k} \leq n$, and positive integers $n_{k}$ such that $\left.f_{k} \equiv f^{n_{1} n_{2} \cdots n_{k}}\right|_{X_{k}}$ maps $X_{k}$ onto $X_{k}$ and $n_{1} \cdots n_{k}$ divides $r$. It must be the case that for some $K, n_{k}=1$ for all $k \geq K$. Thus if $X_{\infty}=\bigcap_{k \geq 1} X_{k}$ then $X_{\infty}$ is an $l_{\infty}$-od for some positive integer $l_{\infty} \leq n,\left.f_{\infty} \equiv f^{n_{1} n_{2} \cdots n_{K}}\right|_{X_{\infty}}$ maps $X_{\infty}$ onto $X_{\infty}$, and in $X_{\infty}$ there is a periodic point of period $p\left(r / n_{1} \cdots n_{K}\right)$ under $f_{\infty}$. 
This process can be continued with transfinite induction as long as the $\left(X_{\alpha}, f_{\alpha}\right)$ are hereditarily decomposable. Since $\left(X_{\alpha}, f_{\alpha}\right)$ is homeomorphic to a subcontinuum of $(X, f)$ and thus is hereditarily decomposable, the process continues indefinitely. Now for $\alpha<\beta, X_{\beta}$ is proper in $X_{\alpha}$ unless $X_{\alpha}$ is a single point. Thus there must be a (countable) ordinal $\alpha$ such that $X_{\alpha}$ is a single point. But $X_{\alpha}$ contains a periodic orbit of period some multiple of $p>1$, and this is not possible. Thus $(X, f)$ must contain an indecomposable subcontinuum.

Theorem 4.3. Suppose that $f: G \rightarrow G$ is a map of the finite graph $G$ and that $h(f)>0$. Then $(G, f)$ contains an indecomposable subcontinuum.

Proof. As in the proof of 4.2, suppose that $(G, f) \equiv\left(G_{0}, f_{0}\right)$ is hereditarily decomposable. Let $\mathscr{G}_{0}$ be the decomposition of $\left(G_{0}, f_{0}\right)$ with quotient map $g:\left(G_{0}, f_{0}\right) \rightarrow \mathscr{G}_{0}$ and $F_{0}: \mathscr{G}_{0} \rightarrow \mathscr{G}_{0}$ the homeomorphism induced by $\hat{f}_{0}$. According to 4.1 , there is a periodic (under $F_{0}$ ) point $\xi \in \mathscr{G}_{0}$ such that $h\left(\hat{f}_{0}, g^{-1}(\xi)\right)>0$. Let $n_{1}$ be the period of $\xi$, let $G_{1}=\Pi_{0}\left[g^{-1}(\xi)\right]$ and let $f_{1}=\left.f_{0}^{n_{1}}\right|_{G_{1}}$. Then $G_{1}$ is a proper subcontinuum of $G_{0}, f_{1}$ maps $G_{1}$ onto $G_{1},\left(G_{1}, f_{1}\right)$ is homeomorphic with $g^{-1}(\xi)$ and hence is hereditarily decomposable, and $h\left(f_{1}\right)=h\left(\hat{f}_{1}\right)=n_{1} h\left(\hat{f}, g^{-1}(\xi)\right)>0$. Continuing in this way we define a strictly decreasing sequence of graphs $G_{0} \supseteq G_{1} \supseteq G_{2} \supseteq \cdots$, positive integers $n_{0}=1, n_{1}, n_{2}, \ldots$, and maps $f_{k}=\left.f^{n_{1} n_{2} \cdots n_{k}}\right|_{G_{k}}: G_{k} \rightarrow G_{k}$ such that $h\left(f_{k}\right)>0$ for all $k$. For each $k \geq 1, G_{k}=\Pi_{0}\left[g^{-1}(\xi)\right]$ where $\xi$ is an element of the decomposition space $\mathscr{G}_{k-1}$ of $\left(G_{k-1}, f_{k-1}\right)$ periodic of period $n_{k}$ under the induced homeomorphism $F_{k-1}: \mathscr{G}_{k-1} \rightarrow \mathscr{G}_{k-1}$. Thus the subcontinua $f_{k-1}^{r}\left(G_{k}\right)$ of $G, r=0, \ldots, n_{k}-1$, are pairwise disjoint and $f^{n_{1} n_{2} \cdots n_{k}}\left(G_{k}\right)=f_{k}\left(G_{k}\right)=G_{k}$.

Now if the sequence $\left\{n_{1} n_{2} \cdots n_{k}\right\}_{k=1}^{\infty}$ is unbounded there must be a $k$ and an $r, 0 \leq r<n_{1} n_{2} \cdots n_{k}$, such that $f_{k}^{r}\left(G_{k}\right)=I$ is an arc ( $G$ is a finite graph), and $\left.f^{n_{1} n_{2} \cdots n_{k}}\right|_{I}: I \rightarrow I$ has positive entropy. But then $f^{n_{1} n_{2} \cdots n_{k}}$ has a horseshoe in $I$ [Mi] and thus by $2.4(G, f)$ has an indecomposable subcontinuum, contrary to our assumption.

Thus the sequence $\left\{n_{1} n_{2} \cdots n_{k}\right\}_{k=1}^{\infty}$ must be bounded and there is a $K$ such that $n_{k}=1$ for all $k \geq K$. Now $G_{K} \supseteq G_{K+1} \supseteq G_{K+2} \cdots$ is a strictly decreasing sequence of finite graphs, each invariant under $f_{K}$. Since $G$ has only finitely many branch points, there are $l$ and $n$ such that for each $s \geq 1, G_{K+l} \backslash G_{K+l+s}$ is a finite disjoint union of half-open arcs $A_{i}^{s}, i=1, \ldots, n$, with $A_{i}^{s} \subseteq A_{i}^{r}$ for $s \leq r$. Let $G_{\infty}=\bigcap_{k=1}^{\infty} G_{k}$. Then $G_{\infty}$ is a subcontinuum of $G$ invariant under $f_{K}$ and $G_{K+l} \backslash G_{\infty}$ is the disjoint union of half-open arcs $A_{i}, i=1, \ldots, n$, where $A_{i}=\bigcup_{s>1} A_{i}^{s}$.

Let $X$ be the quotient space $G_{K+l} / G_{\infty}$ and let $t: X \rightarrow X$ be the map induced by $f_{K+l}: G_{K+l} \rightarrow G_{K+l}$. Note that $X$ is an $n$-od. We consider two cases.

Case 1. $h(t)>0$ : Then $(X, t)$ contains an indecomposable subcontinuum by 4.2. But $\hat{p}:\left(G_{k+l}, f_{k+l}\right) \rightarrow(X, t)$ defined by $\hat{p}\left(x_{0}, x_{1}, \ldots\right)=\left(p\left(x_{0}\right)\right.$, $\left.p\left(x_{1}\right), \ldots\right)$, where $p: G_{K+l} \rightarrow X$ is the quotient map, is a monotone surjection. It follows that $\left(G_{K+l}, f_{K+l}\right)$ must also contain an indecomposable subcontinuum, contrary to the assumption that $(G, f)$ is hereditarily decomposable. (If $H \subseteq(X, t)$ is indecomposable, then $\hat{p}^{-1}(H)$ is a subcontinuum 
of $\left(G_{K+l}, f_{K+l}\right)$ and if $C$ is a continuum in $\hat{p}^{-1}(H)$ irreducible with respect to the property that $\hat{p}(C)=H$, then $C$ is indecomposable.)

Case 2. $h(t)=0$ : In this case, since $p: G_{K+l} \rightarrow X$ semiconjugates $f_{K+l}$ with $t$ and $p$ is one-to-one off $G_{\infty}$, it follows from property (vi) of entropy listed at the beginning of this section that $h\left(\left.f_{K+l}\right|_{G_{\infty}}\right)>0$. Let $f_{\infty}=\left.f_{K+l}\right|_{G_{\infty}}: G_{\infty} \rightarrow$ $G_{\infty}$. Then $h\left(f_{\infty}\right)>0$ and we may inductively define subcontinua $G_{\alpha}$ and positive entropy maps $f_{\alpha}: G_{\alpha} \rightarrow G_{\alpha}$ for all ordinals $\alpha$, as above. But for $\alpha<$ $\beta, G_{\beta}$ is proper in $G_{\alpha}$ unless $G_{\alpha}$ is a single point. Thus for some (countable) ordinal $\alpha, G_{\alpha}$ is a single point. But then $h\left(f_{\alpha}\right)=0$, a contradiction. Thus $(G, f)$ must contain an indecomposable subcontinuum.

\section{BIBLIOGRAPHY}

[AKM] R. L. Adler, A. G. Konheim, and M. H. McAndrew, Topological entropy, Trans. Amer. Math. Soc. 114 (1965), 309-319.

[Ba] M. Barge, The topological entropy of homeomorphisms of Knaster continua, Houston J. Math. 13 (1987), 465-485.

[BM $\left.{ }_{1}\right]$ M. Barge and J. Martin, Chaos, periodicity, and snakelike continua, Trans. Amer. Math. Soc. 289 (1985), 355-364.

$\left[\mathrm{BM}_{2}\right] \longrightarrow$, The construction of global attractors, Proc. Amer. Math. Soc. 110 (1990), 523-525.

[BR] M. Barge and R. Roe, Circle maps and inverse limits, Topology Appl. 36 (1990), 19-26.

[BI] A. M. Blokh, On some properties of graph maps: Spectral decomposition, Misiurewicz conjecture and abstract sets of periods, preprint.

[Bo] R. Bowen, Entropy for group endomorphisms and homogeneous spaces, Trans. Amer. Math. Soc. 153 (1971), 401-510.

[Go] L. W. Goodwyn, Axioms for topological entropy, preprint, 1974.

[He] G. W. Henderson, The pseudo-arc as an inverse limit with one binding map, Duke Math. J. 31 (1964), 421-425.

[Hy] J. Hocking and G. Young, Topology, Dover, 1961.

[Ku] D. P. Kuykendall, Irreducibility and indecomposability in inverse limits, Fund. Math. 80 (1973), 265-270.

[LM] J. Llibre and M. Misiurewicz, Excess of gods implies chaos, preprint.

[Mi] M. Misiurewicz, Horseshoes for mappings of the interval, Bull. Acad. Polon. Sci. Math. 27 (1979), 167-169.

[Ro] R. Roe, Monotone decompositions of inverse limit spaces based on finite graphs, Topology Appl. 34 (1990), 235-245.

[Wi] R. Williams, One-dimensional nonwandering sets, Topology 6 (1967), 473-487.

Department of Mathematics, Montana State University, Bozeman, Montana 59717 29424

Department of Mathematics, College of Charleston, Charleston, South Carolina 